\title{
Protéolyse et texture des fromages à pâte cuite pressée. I. Influence de l'activité de l'eau
}

\author{
A Delacroix-Buchet *, P Trossat ** \\ INRA, station de recherches en technologie et analyses laitières, 39800 Poligny, France
}

(Reçu le 23 décembre 1988; accepté le 31 décembre 1990)

\begin{abstract}
Résumé - Un Gruyère de $45 \mathrm{~kg}$ a été découpé avant salage en 14 petits fromages identiques de $900 \mathrm{~g}$ qui ont été salés en saumure, par paires, durant $0,1,2,4,6,8$ et $24 \mathrm{~h}$. Les petits fromages ont ensuite été affinés 2 semaines à $13{ }^{\circ} \mathrm{C}$ et 4 semaines à $18{ }^{\circ} \mathrm{C}$ sous film plastique. Les différences de temps de saumurage ont permis de faire varier la teneur en $\mathrm{NaCl}$ dans l'humidité des fromages, à 6 semaines, de 0 à $4 \%$. La variation de teneur en $\mathrm{NaCl}$ a induit une variation inverse de l'activité de l'eau des fromages de 0,966 à $0,981(R=-0,965, P<0,001)$ mais n'a pas eu d'incidence sur la teneur en azote soluble dans l'acide phosphotungstique (azote non protéique) des fromages. Les effets de l'activité de l'eau sur la protéolyse et la texture des petits fromages ont été analysés en relation avec la teneur en $\mathrm{NaCl}$ mesurée dans l'humidité des fromages.

Les résultats montrent que l'activité de la plasmine sur la caséine $\beta$ (apparition des fractions $\gamma$ ) augmente jusqu'à une activité de l'eau de 0,972 (soit une teneur en $\mathrm{NaCl}$ dans l'humidité des petits fromages de $2 \%$ ) puis diminue ensuite. La protéolyse de la caséine $\alpha$ s1 décroît avec l'activité de l'eau des fromages : parallèlement, la cohésion de la pâte du fromage mesurée par la déformation à la rupture augmente. La protéolyse secondaire, mesurée par les rapports de l'azote soluble dans l'eau à l'azote total et de l'azote soluble dans l'acide phosphotungstique à l'azote total, varie peu pour les valeurs d'activité de l'eau mesurées.
\end{abstract}

\section{fromage / Gruyère / protéolyse / texture / activité de l'eau}

Summary - Proteolysis and texture of Gruyère type cheese. I. Influence of water activity. One 45-kg loaf of Gruyère cheese was cut before salting into 14 small identical cheeses of $900 \mathrm{~g}$ which were salted in brine in pairs for $0,1,2,4,6,8$ and $24 \mathrm{~h}$. These small cheeses were wrapped in plastic bags and ripened for 2 wk at $13^{\circ} \mathrm{C}$ followed by 4 wk at $18{ }^{\circ} \mathrm{C}$. The differences in brine salting times produced differences in the salt in the moisture content of the 6-wk-old cheeses from $0-4 \%$. The $\mathrm{NaCl}$ content variations led to an opposite variation in the water activity of the cheeses from $0.966-0.981(R=-0.965, \mathrm{P}<0.001)$ and had no influence on the phosphotungstic acid-soluble nitrogen (non protein nitrogen) content of the cheese. Proteolysis and texture of the cheeses were analysed in relation to their water activity. Results showed that the plasmin activity on $\beta$-casein (appearance of $\gamma$ fractions) increased up to a water activity of 0.972 (ie salt in moisture content of $2 \%$ ), followed by a regular decrease. The $\alpha$ s1 casein breakdown decreased with cheese water activity, lowering the cohesiveness of the cheese. Secondary proteolysis, as measured by the ratios watersoluble $N /$ total $N$ and phosphotungstic acid-soluble $N /$ total $N$, varied little between the water activity values measured in the cheeses.

\section{cheese / Gruyère / proteolysis / texture / water activity}

\footnotetext{
* Adresse actuelle : INRA, station de recherches laitières, 78350 Jouy-en-Josas, France

** Stagiaire de DEA. Université de Franche-Comté, Besançon, France. Adresse actuelle : Institut technique de Gruyère, 70190 Rioz, France
} 


\section{INTRODUCTION}

Au cours de l'affinage, les caséines, constituants principaux du fromage, subissent une dégradation enzymatique due à l'action cumulée des enzymes natives du lait, des enzymes coagulantes (présure) et des enzymes bactériennes (levain et psychrotrophes). Cette protéolyse est déterminante dans le développement de la texture et de l'arôme caractéristique des fromages (Adda et al, 1982).

La part respective de chacune de ces enzymes ou systèmes enzymatiques, dans la qualité finale du fromage, dépend de nombreux paramètres de fabrication et d'affinage. Les paramètres de fabrication déterminent les cinétiques de coagulation, d'acidification et d'égouttage du caillé qui aboutissent suivant les technologies employées à des valeurs caractéristiques de $\mathrm{pH}$ et de proportion de caséines dans l'humidité des fromages au démoulage (Lawrence et al, 1987). Ils interviennent également dans les quantités d'enzymes résiduelles présentes dans le caillé. Les conditions d'affinage (salage des fromages, température et humidité des caves, présence ou non d'une flore de surface) modifient ensuite les paramètres du caillé tels que $\mathrm{pH}$, Eh et activité de l'eau (Aw) (Adda et al, 1982) dont dépend étroitement l'activité des enzymes.

Très peu d'études ont été réalisées sur l'activité de l'eau des fromages (Ruegg et Blanc, 1977; Mathlouthi et al, 1980; Marcos et al, 1981) alors que les processus physico-chimiques de l'affinage sont conditionnés par la disponibilité de l'eau dans ce type de milieu. Ruegg et Blanc (1977) et Marcos et al (1981) ont montré que l'activité de l'eau d'un fromage est surtout déterminée par sa teneur en eau mais aussi sa teneur en constituants de faible poids moléculaire $(\mathrm{NaCl}$, azote non protéique) et son $\mathrm{pH}$.
Le salage du fromage peut être considéré comme une technique susceptible d'abaisser l'activité de l'eau (Hardy, 1979).

L'influence du chlorure de sodium sur la protéolyse des caséines a été plus largement étudiée. Cependant, si de nombreux travaux ont été réalisés sur l'activité des protéases en milieu salin (Fox et Walley, 1971; Sanogo et al, 1987), peu de travaux ont été menés dans des milieux salés aussi complexes que le fromage. À notre connaissance, seuls existent des travaux sur Cheddar (Phelan et al, 1973; Thomas et Pearce, 1981), sur Meshanger (Noomen, 1978a, b) et sur Bleu (Godinho et Fox, 1982).

Un essai a donc été conduit pour connaître l'influence de l'Aw d'un fromage type Gruyère sur sa protéolyse et sa texture en induisant des variations de l'Aw par des variations de la teneur en $\mathrm{NaCl}$ des fromages.

\section{MATÉRIEL ET MÉTHODES}

\section{Protocole expérimental}

L'objectif de l'essai étant de mesurer l'incidence de l'activité de l'eau (Aw) sur la protéolyse et la texture d'un fromage à pâte cuite pressée, un Gruyère de $45 \mathrm{~kg}$ a été découpé, avant salage, en petits fromages de $900 \mathrm{~g}$, salés ensuite à différentes teneurs en chlorure de sodium $(\mathrm{NaCl})$.

Le fromage initial a été fabriqué selon une technologie classique, à partir de lait cru, ensemencé avec $1 \%$ de streptocoques thermophiles, $0,6 \%$ de lactobacilles thermophiles et coagulé avec $0,18 \%$ d'extrait de présure liquide à 800 $\mathrm{mg}$ de chymosine active par litre. Après pressage, le fromage âgé de $20 \mathrm{~h}$ a été découpé en petits fromages. Pour cela, 7 cylindres ont été prélevés à l'emporte-pièce, sur un même cercle situé à environ mi-rayon du fromage. Après élimination des extrémités de chaque cyclindre, 2 fromages ont été découpés dans leur hauteur. Nous avons ainsi obtenu 14 petits fromages 
identiques, homogènes dans leur composition, de forme cylindrique, d'un diamètre de $15 \mathrm{~cm}$ et d'une hauteur en talon de $3,5 \mathrm{~cm}$. Deux des petits fromages n'ont pas été salés, les 12 autres, répartis en 6 paires, ont été salés dans une saumure saturée en $\mathrm{NaCl}$, à $13^{\circ} \mathrm{C}$ et $\mathrm{pH} 5,20$, respectivement $1,2,4,6,8$ et $24 \mathrm{~h}$.

Pour tenir compte de la taille réduite des fromages et empêcher tout développement d'une flore à la surface des fromages, les 14 petits fromages ont été traités en surface par une pulvérisation d'antifongique (pyramicine) et affinés, emballés sous film de polyéthylène semiperméable, 2 semaines à $13^{\circ} \mathrm{C}$ et 4 semaines à $18^{\circ} \mathrm{C}$.

\section{Méthodes}

\section{Analyses chimiques}

Les analyses de fromage à $20 \mathrm{~h}$ ont été réalisées dans les chutes, situées à mi-rayon du fromage ayant servi à la découpe des petits fromages. Ces derniers ont été analysés à 6 semaines à partir d'un secteur prélevé sur chacun d'eux.

- $\mathrm{pH}$ (20 h et 6 semaines) : après broyage du fromage frais, mesure avec une électrode combinée placée en contact avec le fromage tassé dans un godet. La mesure est relevée lorsque l'équilibre est atteint.

Les analyses effectuées portent sur :

- extrait sec ( $E S, 20$ h et 6 semaines) : dosage selon la norme FIL 4A (1982);

- matière grasse (MG, $20 \mathrm{~h}$ ) : méthode butyrométrique de Heiss (1961);

- calcium (20 h et 6 semaines) ; dosage complexométrique selon la technique de Pearce (1977);

- chlorure de sodium ( $\mathrm{NaCl}, 20 \mathrm{~h}$ et 6 semaines) : mesure réalisée à l'aide d'un chloruremètre (Corning 926) à électrode soluble d'argent, étalonné par rapport à la technique de dosage des chlorures préconisée dans la norme FIL 17A (1972);

- activité de l'eau ( $A w, 6$ semaines) : méthode par interpolation graphique de Landrock et Proctor (1951);

- indice d'affinage (IA, 6 semaines) : méthode d'appréciation rapide du stade d'affinage des fromages par la technique cryoscopique de Courroye (1987);

- azote total ( $20 \mathrm{~h}$ et 6 semaines) et fractions azotées (6 semaines) : dosage par la méthode Kjeldahl de l'azote total (NT), de l'azote soluble dans l'eau (NS) après préparation selon la méthode de Kuchroo et Fox (1982) et de l'azote soluble dans l'acide phosphotungstique (NPT) après préparation selon la technique de Gripon et al (1975);

- fractions caséines (6 semaines) : analyse quantitative des principales caséines et de leurs produits de dégradation ( $\alpha$ s1, $\beta, \alpha$ s1-1, $\beta$ dégradées, $\gamma 1, \gamma 2$ et $\gamma 3$ ) après séparation électrophorétique des caséines et lecture densitométrique des plaques d'électrophorèse selon la technique décrite par Buchet (1987). La caséine entière est préparée en dissolvant dans $5 \mathrm{ml}$ d'urée 9 mol. $1^{-1}$ contenant $1 \%$ de $\beta$-mercaptoéthanol, $0,3 \mathrm{~g}$ du culot de centrifugation obtenu lors de la préparation de l'azote soluble dans l'eau (NS). Les différentes caséines sont ensuite séparées sur gel PAGE et leurs pourcentages dans la caséine totale déterminés selon la technique décrite par Collin et al (1987).

\section{Tests rhéologiques}

La texture des fromages affinés a été appréciée par 2 types de tests.

\section{Test d'extrusion}

Ce test a été réalisé à $20^{\circ} \mathrm{C}$ sur un cylindre de fromage d'environ $1 \mathrm{~cm}$ de hauteur sur $1 \mathrm{~cm}$ de diamètre prélevé avec la sonde de l'appareil. La force d'extrusion $(\mathrm{kg})$ a été mesurée avec un extrudeur type Fira Nird Extruder équipé d'une filière de $2 \mathrm{~mm}$ de diamètre. C'est un indicateur de la fermeté du fromage.

\section{Test de compression simple uniaxiale destructive}

Les tests de compression ont été effectués dans une enceinte thermostatée avec le rhéomètre digital INRA (brevet $\mathrm{Fr} n^{\circ}$ 8514870) équipé d'un capteur de $500 \mathrm{~N}$. Les échantillons ont été prélevés sur toute la hauteur du fromage à l'aide d'un emporte-pièce de diamètre interne $20 \mathrm{~mm}$ puis on a éliminé symétriquement les parties externes de façon à obtenir des carottes cylindriques d'environ $2 \mathrm{~cm}$ de hauteur. 
Les mesures ont été réalisées en double à $18^{\circ} \mathrm{C}$. Les éprouvettes ont été comprimées à vitesse constante $(30 \mathrm{~mm} / \mathrm{min})$ jusqu'à un niveau de compression de $80 \%$ susceptible d'entraîner la rupture de l'éprouvette.

Par représentation graphique des résultats, on obtient une courbe dite de compression à partir de laquelle on détermine plusieurs paramètres (fig 1) :

- le module d'élasticité (E) ou module de Young, qui est la pente de la courbe à l'origine, représente l'élasticité du fromage. Cette valeur est déterminée dans la partie linéaire de la courbe (domaine d'élasticité linéaire) pour des déformations de l'ordre de $5 \%$;

- la contrainte au point de rupture (Ef) (en $\mathrm{N} / \mathrm{m}^{2}$ ou $\mathrm{Pa}$ ) est obtenue au point d'inflexion de la courbe. Ce paramètre constitue un indicateur de la fermeté du fromage;

- la déformation au point de rupture $\left(\varepsilon_{f}\right)$ calculée selon Cauchy (en \%) $\left(\Delta H / H_{0}\right)$. Cette valeur constitue un indicateur de la cohésion du fromage;

- le travail $(\omega)$ (en $\mathrm{J}$ ) réalisé pour comprimer le fromage à $80 \%$, représenté par l'aire sous la courbe jusqu'à la rupture.

\section{Analyses statistiques}

Pour étudier les liaisons entre variables, nous avons procédé par étapes.

Tout d'abord, nous avons calculé la matrice de corrélations simples entre variables pour l'ensemble des données analytiques. Lorsque les coefficients de corrélations simples entre 2 variables étaient significatifs, nous avons en-

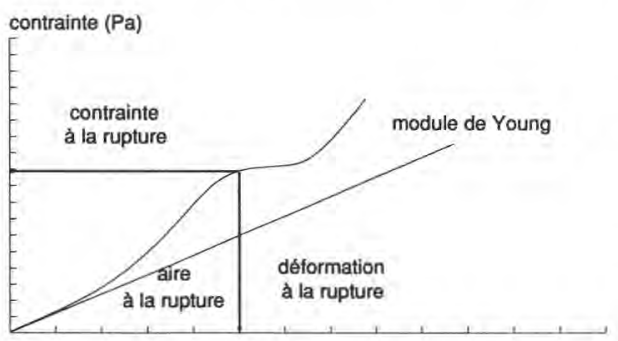

déformation (p.100)

Fig 1. Courbe de compression. Compression curve. suite calculé les régressions linéaires simples correspondantes. Enfin, lorsqu'il nous a paru intéressant d'analyser simultanément un ensemble de variables, nous avons fait appel à une méthode d'analyse statistique multidimensionnelle : l'analyse en composantes principales (ACP). Dans notre étude, nous nous sommes inspirés de l'article de Fils-Lycaon et al (1988) pour caractériser, à l'aide de l'ACP, l'évolution des profils protéolytiques des fromages en fonction de leur $A w$.

Les analyses statistiques ont été réalisées sur micro-ordinateur à partir du logiciel Microstat, élaboré par l'ITCF (1984).

\section{RÉSULTATS ET DISCUSSION}

\section{Composition physico-chimique}

Le fromage à $20 \mathrm{~h}$ a un extrait-sec (ES) égal à $63,61 \%$ de son poids et un $\mathrm{pH}$ de 5,18 . Ses teneurs en matière grasse, en protéine et en calcium sont respectivement de $47,70,46,51$ et $1,58 \mathrm{~g} / 100 \mathrm{~g}$ d'ES, valeurs normales pour un Gruyère de Comté.

La composition moyenne et la variabilité de composition des 14 petits fromages âgés de 6 semaines sont données dans le tableau I.

II apparaît que le $\mathrm{pH}$ moyen augmente environ de 0,3 unité et l'ES de $0,7 \mathrm{~g} / 100 \mathrm{~g}$ de fromage au cours des 6 semaines d'affinage. Pendant la même période d'affinage de 6 semaines, Berdagué et Grappin (1987) observent une augmentation comparable du $\mathrm{pH}$ moyen de 96 gruyères de Comté $(+0,38$ unité) mais très supérieure de l'ES (+2,3 g/100 $\mathrm{g}$ de fromage). L'emballage des petits fromages a donc très fortement diminué les pertes d'eau par évaporation, objectif qui était recherché.

Les teneurs en $\mathrm{NaCl}$ des petits fromages varient de 0,05 à $1,41 \mathrm{~g} / 100 \mathrm{~g}$ en fonction des temps de saumurage pratiqués. La cinétique d'absorption du sel 
Tableau I. Composition physico-chimique et texture (moyenne et écart type) des fromages de 6 semaines.

Physico-chemical composition and texture (mean and standard deviation) of 6-wk-old cheeses.

\begin{tabular}{|c|c|c|c|c|}
\hline $\begin{array}{l}\text { VARIABLES } \\
\text { variables }\end{array}$ & $\begin{array}{l}\text { MOYENNE ET } \\
\text { ERREUR-TYPE } \\
\text { mean and } \\
\text { standard error }\end{array}$ & $\begin{array}{l}\text { MINIMUM- } \\
\text { MAXIMUM } \\
\text { min-max }\end{array}$ & $\begin{array}{l}\text { ECART- } \\
\text { TYPE } \\
{ }^{*} S D\end{array}$ & $\begin{array}{c}\text { COEFFICIENT } \\
\text { DE VARIATION } \\
(\mathrm{p} .100) \\
(\mathrm{SD} / \text { mean }) \times 100\end{array}$ \\
\hline$\underset{p H}{\mathrm{pH}}$ & $5,49 \pm 0,01$ & $5,40-5,56$ & 0,05 & 0,8 \\
\hline $\begin{array}{l}\text { extrait sec } \\
\text { dry matter }\end{array}$ & $64,29 \pm 0,20$ & $63,52-66,59$ & 0,74 & 1,2 \\
\hline $\begin{array}{l}\text { chlorure de sodium (1) } \\
\text { sodium chloride }\end{array}$ & $0,65 \pm 0,11$ & $0,05-1,41$ & 0,42 & 63,9 \\
\hline $\begin{array}{l}\text { sel dans l'humidité (2) } \\
\text { salt in moisture }\end{array}$ & $1,85 \pm 0,32$ & $0,14-4,08$ & 1,22 & 65,7 \\
\hline $\begin{array}{l}\text { activité de l'eau } \\
\text { water activity }\end{array}$ & $0,973 \pm 0,001$ & $0,966-0,981$ & 0,004 & 0,4 \\
\hline $\begin{array}{l}\text { calcium } \\
\text { calcium }\end{array}$ & $1,56 \pm 0,01$ & $1,47-1,61$ & 0,04 & 2,6 \\
\hline $\begin{array}{l}\text { azote total (NT) } \\
\text { total nitrogen (TN) }\end{array}$ & $46,34 \pm 0,11$ & $45,83-47,06$ & 0,39 & 0,9 \\
\hline $\begin{array}{l}{ }^{* *} \mathrm{NS} / \mathrm{NT} \\
W S N / T N\end{array}$ & $11,09 \pm 0,20$ & $9,19-11,87$ & 0,74 & 6,6 \\
\hline $\begin{array}{l}{ }^{* * *} \text { NPT/NT } \\
P T A S N / T N\end{array}$ & $5,80 \pm 0,15$ & $4,91-6,75$ & 0,55 & 9,5 \\
\hline $\begin{array}{l}\text { caséines } \gamma \\
\gamma \text { caseins }\end{array}$ & $15,84 \pm 0,99$ & $9,52-21,86$ & 3,71 & 23,4 \\
\hline $\begin{array}{l}\text { caséine } \alpha_{\mathrm{s} 1-\mathrm{I}} \\
\alpha_{\mathrm{s} 1-\mathrm{I}} \text { casein }\end{array}$ & $9,69 \pm 0,63$ & $5,98-13,00$ & 2,36 & 24,3 \\
\hline $\begin{array}{l}\text { indice d'affinage (5) } \\
\text { ripening index }\end{array}$ & $0,171 \pm 0,01$ & $0,115-0,259$ & 0,040 & 24,3 \\
\hline
\end{tabular}

(1) $\mathrm{g} / 100 \mathrm{~g}$ de fromage ( $\mathrm{g} / 100 \mathrm{~g}$ of cheese). (2) p 100 (percent). (3) g/100 g d'extrait sec ( $g / 100 \mathrm{~g}$ of dry matter). (4) p 100 des caséines totales (percent of whole casein). (5) degré Celsius (degree Celsius). (6) kg.

"SD : standard deviation. "* NS : azote soluble dans l'eau (WSN: water-soluble nitrogen). "** NPT: azote soluble dans l'acide phosphotungstique (PTASN: phosphotungstic acid-soluble nitrogen).

dans les fromages de petit format est comparable à celle observée lors du salage de l'Emmental (Chamba, 1988). En effet, l'accroissement de la teneur en $\mathrm{NaCl}$ des minifromages est bien corrélé à la racine car- rée de la durée du saumurage exprimée en $\mathrm{h}(R=+0,992, P<0,001)$. La relation est linéaire jusqu'à $8 \mathrm{~h}$ de saumurage (fig 2). Le calcul de répétabilité (norme AFNOR NF X06041, 1970) de cette techni- 


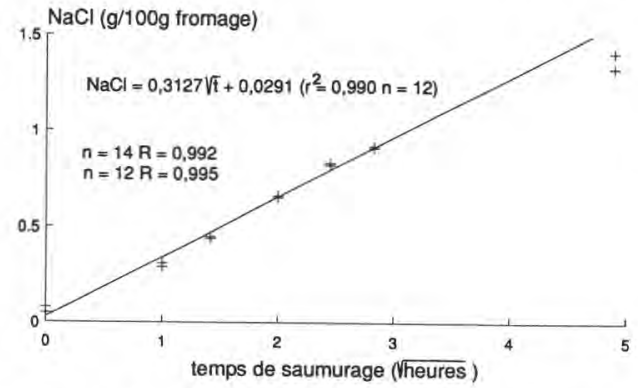

Fig 2. Cinétique d'absorption du sel par les Gruyères.

Kinetics of salt absorption by Gruyère type cheeses.

que de saumurage a également retenu toute notre attention. L'écart type de répétabilité $s_{r}$, la répétabilité $r$ (exprimée par l'écart maximal obtenu entre doubles, $r=$ $2,77 \times S_{r}$ pour $P<0,95$ ) et le coefficient de variation en fonction de la moyenne des valeurs obtenues $\left(C V_{r}=\left(S_{r} / X\right) \times 100\right)$ sont respectivement de $0,024 \mathrm{~g} / 100 \mathrm{~g}$ de fromage, $0,067 \mathrm{~g} / 100 \mathrm{~g}$ de fromage et $3,7 \%$ de la moyenne des taux de sel mesurés. On peut donc estimer qu'en saumurant des minifromages a priori identiques, on obtient pour un temps donné de saumurage des valeurs reproductibles de la teneur en sel de ces fromages.

Le saumurage entraînant une perte d'eau des fromages (Chamba, 1988) l'ES des fromages augmente avec leur teneur en $\mathrm{NaCl}$ de 63,5 à $66,2 \mathrm{~g} / 100 \mathrm{~g}(R=+$ $0,869, P<0,001$ ).

L'activité moyenne de l'eau mesurée dans les petits fromages $(A w=0,973)$ est proche de celle mentionnée par Ruegg et Blanc (1977) dans le Gruyère. La mesure de l'Aw par la méthode de Landrock et Proctor (1951) semble suffisamment précise pour intégrer des variations du taux de $\mathrm{NaCl}$ dans l'humidité des fromages de $0-4 \%$ : la corrélation entre l'Aw et la teneur en $\mathrm{NaCl}$ dans l'humidité des petits fro- mages est très hautement significative ( $R=-0,965 ; P<0,001$ ) (fig 3 ).

La corrélation entre l' $A w$ et l'humidité du fromage est également très élevée $(R=$ $+0,831, P<0,001)$.

On n'observe pas de relation linéaire entre l' $A w$ des fromages et leur $\mathrm{pH}$ mais une augmentation du $\mathrm{pH}$ jusqu'à une $A w$ proche de 0,975 puis une diminution. Marcos et al (1981) n'observent pas non plus de corrélation significative entre le $\mathrm{pH}$ et l' $A w$ de différents fromages.

Pour discuter les résultats sur la protéolyse, il semble donc possible d'utiliser les valeurs d'Aw. Ces valeurs prennent en cumpte à la fois la concentration en sels dissous et l'humidité du fromage. Elles sont par conséquent plus intéressantes que les seuls valeurs de teneur en $\mathrm{NaCl}$.

\section{Protéolyse primaire}

La protéolyse primaire a été mesurée par l'apparition des fractions $\alpha s 1-\mid$ et $\gamma$ issues de l'hydrolyse respective des caséines $\alpha$ s1 et $\beta$.

Pour des Gruyères âgés de 6 semaines, la proportion de fraction $\alpha s 1-1$

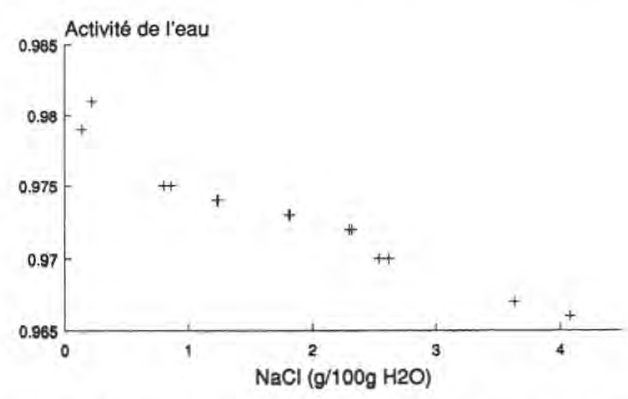

Fig 3. Influence de la teneur en sel dans l'humidité sur l'activité de l'eau des gruyères.

Effect of salt in moisture content on water activity of Gruyère-type cheeses. 
dans la caséine totale est dans notre étude très inférieure à la proportion moyenne trouvée dans des Gruyères de Comté du même âge par Berdagué et Grappin (1987) $(9,7$ vs $25,6 \%)$. Cependant, ces auteurs rapportent que cette proportion est très variable entre fromages $(13,7$ à $34,1 \%$ de la caséine totale). À âge identique des fromages, la proportion moyenne des fractions $\gamma$ dans la caséine totale n'est, par contre, que légèrement inférieure, dans notre étude, à celle mentionnée par ces 2 mêmes auteurs $(15,8$ vs $18,3 \%)$.

La dégradation de la caséine $\beta$ en fractions $\gamma$ spécifiques de l'action de la plasmine est fortement influencée par l'Aw des fromages (fig 4). Elle augmente dans un premier temps avec la baisse d'Aw induite par l'accroissement de la teneur en $\mathrm{NaCl}$ puis diminue très fortement ensuite. Le maximum de production de fractions $\gamma$ est observé pour une $A w$ voisine de 0,972 qui correspond à une teneur en $\mathrm{NaCl}$ des fromages de $0,8 \%$ (soit $2,3 \%$ dans l'humidité). Ce résultat obtenu pour des fromages type Gruyère est tout à fait comparable à celui trouvé par Noomen (1978a) pour des fromages à pâte molle type Meshanger pour lesquels l'optimum d'activité "plasmine" se situait autour de $2 \%$ de sel

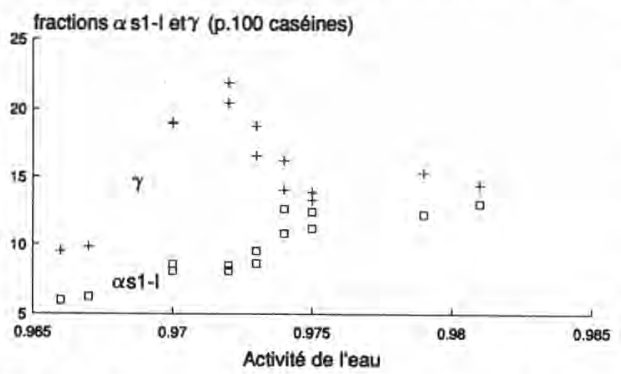

Fig 4. Influence de l'activité de l'eau sur la dégradation des caséines $\beta$ et $\alpha$ s1 exprimée en pourcent de fractions $\gamma(+)$ et $\alpha s 1-1$ (o) dans la caséine totale.

Effect of water activity on $\beta$ - and $\alpha$ s1 casein breakdown expressed as percent of $\gamma(+)$ and $\alpha$ s 1-I (o) fractions of whole casein. dans l'humidité du fromage. Cette teneur en sel peut correspondre à l'optimum d'activité de l'enzyme ou à l'optimum de son activation. Entre des valeurs d'Aw de 0,972 et 0,966 , la proportion de fractions $\gamma$ dans les caséines totales chute de moitié soit d'une valeur proche de $21,0 \%$ à une valeur inférieure à $10,0 \%$. Les fromages ayant une $A w$ de 0,967 contiennent 4,0\% de $\mathrm{NaCl}$ dans l'humidité et ont une activité "plasmine", mesurée par l'apparition des fractions $\gamma$, inférieure à celle des fromages non salés.

La dégradation de la caséine $\alpha$ s 1 en fragment $\alpha s 1-1$ décroît sans cesse avec l'Aw, pour la plage de variation de la teneur en $\mathrm{NaCl}$ étudiée dans nos fabrications fromagères $(R=+0,901, P<0,001)$ (fig 4). Thomas et Pearce (1981) obtiennent un résultat similaire dans du Cheddar. Ils montrent qu'en établissant à travers des blocs de Cheddar un gradient de $\mathrm{NaCl}$ entre 4 et $8 \%$ de sel dans l'humidité, le pourcentage de caséine $\alpha s 1$ dégradée diminue linéairement avec l'augmentation de la teneur en $\mathrm{NaCl}$. Ce pourcentage passe respectivement de 95 à $40 \%$ pour 4 et $8 \%$ de $\mathrm{NaCl}$ après 1 mois d'affinage à $10^{\circ} \mathrm{C}$. Noomen (1978b) observe aussi une diminution de la protéolyse de la caséine $\alpha s 1$ par la présure dans des fromages à pâte molle type Meshanger ayant des teneurs en $\mathrm{NaCl}$ supérieures à $4 \%$ dans l'humidité. Fox et Walley (1971) et Sanogo et al (1987) mesurent un maximum de dégradation de la caséine $\alpha s 1$ par la chymosine dans des solutions salines de caséine purifiée contenant de $5-10 \%$ de $\mathrm{NaCl}$. De telles concentrations en $\mathrm{NaCl}$ ne sont jamais rencontrées dans les Gruyères. D'autre part, il semble peu vraisemblable que l'apparition du fragment $\alpha s 1-$ I soit liée à la seule activité de la chymosine résiduelle dans les Gruyères, après chauffage du lait en cuve à $52-54{ }^{\circ} \mathrm{C}$ (Matheson, 1981; Garnot et Mollé, 1987). On peut penser qu'elle est plutôt due à l'activité enzy- 
matique des microorganismes : les lactobacilles thermophiles hydrolysent partiellement les caséines $\alpha s 1$ et $\beta$ (El Soda et Desmazeaud, 1982; Zevaco et Gripon, 1988 ) et les bactéries propioniques possèdent une activité caséolytique (Searles et al, 1970). Un rôle éventuel de la protéase acide du lait n'est également pas à rejeter comme le suggère Noomen (1978a) dans le fromage à pâte molle type Meshanger. Cette enzyme, stable à la chaleur, est capable de résister à l'étape du chauffage du caillé en cuve et elle dégrade la caséine $\alpha s 1$ pour former des fragments $\alpha s 1-\mathrm{I}$ (Kaminogawa et al, 1980).

\section{Protéolyse secondaire}

La protéolyse secondaire a été mesurée globalement par les teneurs en azote de la fraction azotée soluble dans l'eau (NS) qui représente les polypeptides d'un poids moléculaire jusqu'à $10000 \mathrm{Da}$ et les acides aminés, et de la fraction azotée soluble dans l'acide phosphotungstique (NPT) qui regroupe les petits peptides et les acides aminés.

Les valeurs de NS exprimées en \% de l'azote total sont un peu inférieures à la moyenne des valeurs mesurées par Collin

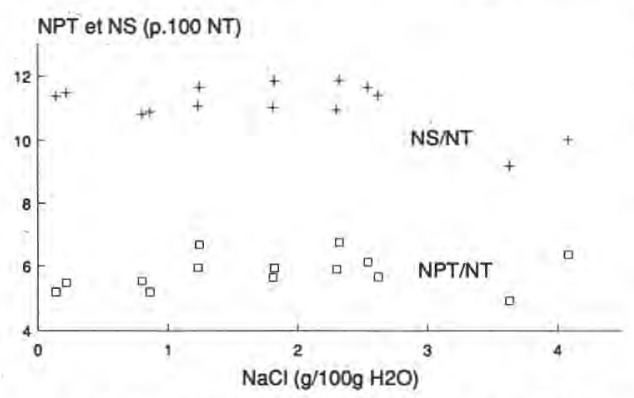

Fig 5. Influence de la teneur en sel dans l'humidité sur la protéolyse secondaire des gruyères. Effect of salt in moisture content on secondary proteolysis in Gruyère-type cheeses. et al (1987) dans des Gruyères de Comté âgés de 6 semaines (11,09 vs 15,55\%). Ceci peut être lié à la plus faible dégradation de la caséine $\alpha s 1$ observée dans nos minifromages, elle même, peut-être, en partie liée, à une teneur moyenne en sel dans l'humidité des minifromages saumurés supérieure à celle des Gruyères de Comté emmorgés $(1,85$ vs $1,57 \mathrm{~g} / 100 \mathrm{~g}$ $d^{\prime} \mathrm{H}_{2} \mathrm{O}$ ). Les valeurs de NPT dans l'azote total sont, par contre, du même ordre de grandeur dans les 2 études $(5,00$ vs $4,85 \%)$.

La protéolyse secondaire semble peu influencée par une augmentation de la teneur en $\mathrm{NaCl}$ dans l'humidité des fromages de 0 à $4 \%$ (fig 5 ). On remarque cependant une diminution de la proportion du NS dans l'azote total des fromages contenant $4,0 \%$ de $\mathrm{NaCl}$ dans l'humidité c'est-àdire ayant une $A w$ inférieure à 0,970. Par contre, il n'existe aucune relation significative entre l' $A w$ et la teneur en NPT exprimée dans l'humidité du fromage $(R=-$ 0,470 ). La variation d'Aw mesurée est donc bien le fait essentiel de celle de la teneur en $\mathrm{NaCl}$ dans l'humidité du fromage, objectif qui était recherché.

L'indice d'affinage des fromages mesuré par la technique cryoscopique est de manière très hautement significative influencé par le taux de $\mathrm{NaCl}$ des fromages $(R=$ $+0,977, P<0,001$ ) (fig 6). Ce résultat revient à dire que, pour des fromages affinés de façon identique et ayant le même âge, l'indice d'affinage des fromages les plus salés $(4,0 \%$ de $\mathrm{NaCl}$ dans l'humidité) qui sont aussi, d'après les résultats énoncés précédemment, les moins protéolysés, est supérieur à celui des fromages moins salés et globalement plus protéolysés. L'étude de Courroye (1987) montre également que cette technique cryoscopique n'est pas satisfaisante pour des fromages dont les niveaux de protéolyse sont très voisins. Sur 180 Emmentals affinés de 


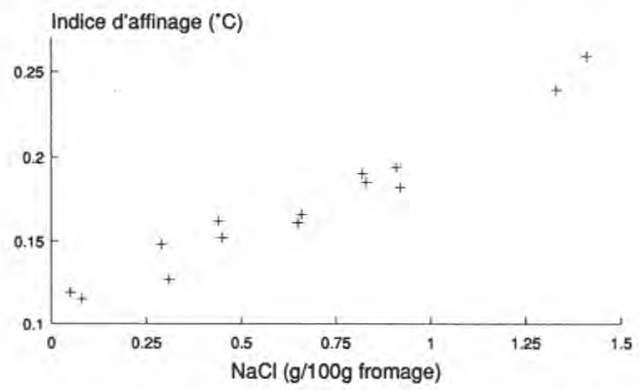

Fig 6. Relation entre l'indice d'affinage et la teneur en sel dans l'humidité des Gruyères.

Relationship between the ripening index and the salt in moisture content of Gruyère-type cheeses.

façon identique et provenant d'une même fromagerie, il obtient une corrélation faible mais significative entre la teneur en $\mathrm{NaCl}$ et l'indice d'affinage des fromages. Nos résultats montrent que l'indice d'affinage ne peut être utilisé par les industriels pour déterminer rapidement le degré de protéolyse des fromages à pâte cuite pressée qu'à condition d'appliquer des corrections en fonction de la teneur en $\mathrm{NaCl}$ de l'échantillon analysé.

\section{Texture}

Les valeurs moyennes obtenues pour les tests rhéologiques sont présentées dans le tableau II. L'élasticité des fromages mesurée par le module de Young et la fermeté évaluée par la contrainte à la rupture sont les paramètres les plus variables.

Les calculs de corrélations simples montrent une liaison très hautement significative $(P<0,001)$ positive entre la contrainte et la déformation à la rupture ( $R$ $=+0,867$ ) et négative entre le module de Young et la déformation à la rupture $(R=$ $-0,843)$.
Les mesures rhéologiques sont elles aussi fortement influencées par l'Aw des fromages. La force d'extrusion, qui permet d'apprécier d'une certaine manière, la fermeté du fromage, augmente en moyenne quand I'Aw diminue $(R=-0,612, P<0,01)$. Cette augmentation est surtout sensible à partir d'une $A w$ de 0,970 soit $2,50 \%$ de $\mathrm{NaCl}$ dans l'humidité.

On observe également de très bonnes corrélations entre les paramètres mesurés lors des tests de compression et l'Aw des minifromages. Les meilleures corrélations sont obtenues avec la déformation à la rupture, qui représente la cohésion de la pâte des fromages $(R=+0,922, P<$ 0,001 ) (fig 7) et la contrainte à la rupture $(R=+0,823, P<0,001)$ (fig 8 ). En saumurant des cylindres prélevés dans des $\mathrm{Ca}$ memberts non affinés, Mpagana et Hardy (1986) observent aussi une augmentation de la fermeté (force au point de rupture) et de la friabilité du fromage (déformation au point de rupture) avec l'augmentation de sa teneur en sel. lls expliquent ces modifications rhéologiques par une diminution de I'hydratation des caséines et une légère augmentation de la viscosité de la phase aqueuse du fromage lorsque sa teneur en sel croît. D'autre part, les fromages pré-

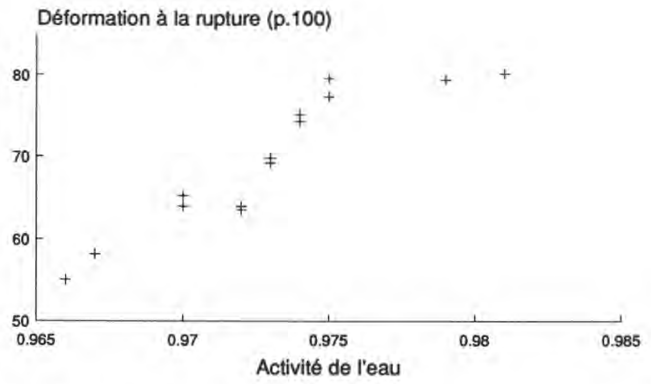

Fig 7. Relation entre la déformation à la rupture (cohésion) et l'activité de l'eau des fromages.

Relationship between the fracture strain (cohesiveness) and water activity in Gruyere-type cheeses. 
Tableau II. Paramètres rhéologiques (moyenne et écart type) des fromages de 6 semaines. Rheological parameters (mean and standard deviation) of 6-wk-old cheese.

\begin{tabular}{|c|c|c|c|c|}
\hline $\begin{array}{l}\text { VARIABLES } \\
\text { variables }\end{array}$ & $\begin{array}{l}\text { MOYENNE ET } \\
\text { ERREUR-TYPE } \\
\text { mean and } \\
\text { standard error }\end{array}$ & $\begin{array}{l}\text { MINIMUM- } \\
\text { MAXIMUM } \\
\text { min-max }\end{array}$ & $\begin{array}{c}\text { ECART- } \\
\text { TYPE } \\
{ }^{*} S D\end{array}$ & $\begin{array}{c}\text { COEFFICIENT } \\
\text { DE VARIATION } \\
(\mathrm{p} .100) \\
(S D / \text { mean }) \times 100\end{array}$ \\
\hline $\begin{array}{l}\text { force d'extrusion (1) } \\
\text { extrusion force }\end{array}$ & $4,85 \pm 0,14$ & $3,80-6,00$ & 0,54 & 11,1 \\
\hline $\begin{array}{l}\text { module de Young (2) } \\
\text { modulus of Young }\end{array}$ & $316 \pm 28$ & 174- 518 & 106 & 33,6 \\
\hline $\begin{array}{l}\text { contrainte à } \\
\text { la rupture } \\
\text { fracture stress }\end{array}$ & $697 \pm 61$ & $457-1149$ & 227 & 32,6 \\
\hline $\begin{array}{l}\text { déformation à } \\
\text { la rupture } \\
\text { fracture strain }\end{array}$ & $69,6 \pm 2,2$ & $55,1-80,1$ & 8,2 & 11,8 \\
\hline $\begin{array}{l}\text { travail à } \\
\text { la rupture } \\
\text { fracture force }\end{array}$ & $0,665 \pm 0,031$ & $0,509-0,870$ & 0,115 & 17,2 \\
\hline
\end{tabular}

(1) kgF. (2) kPa. (3) p 100 (percent). (4) Joules. *SD : écart type (standard deviation).

sentant une Aw élevée sont aussi les fromages possédant le plus faible ES et de nombreux auteurs soulignent l'effet ramollissant de l'eau sur la texture des fromages

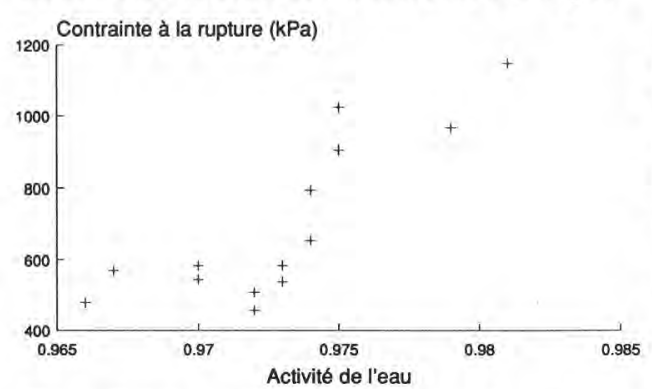

Fig 8. Relation entre la contrainte à la rupture (fermeté) et l'activité de l'eau des fromages.

Relationship between the fracture stress (firmness) and water activity in Gruyère-type cheeses.
(Mocquot, 1979; Ruegg et al, 1980; Eberhard, 1985).

Nous notons aussi une corrélation importante $(R=+0,967, P<0,001)$ entre la proportion de fraction $\alpha$ s1-I dans la caséine totale et la déformation à la rupture. Cette relation résulte probablement de l'influence de l'Aw sur la dégradation de la caséine $\alpha s 1$, déjà décrite dans les résultats. Pour des fromages à pâte molle, De Jong (1977) a montré que le ramollissement de leur pâte dépend directement de la dégradation de la caséine $\alpha s 1$ et augmente avec elle. De même, Creamer et Olson (1982) notent une bonne corrélation entre la quantité de caséine $\alpha s 1$ intacte dans le Cheddar et la contrainte à la rupture des fromages. Dans nos petits fromages, la force d'extrusion est également inverse- 
ment liée à la proportion de fraction $\alpha$ s 1 -I dans la caséine totale $(R=-0,715, P<$ 0,01 ). Elle diminue aussi quand le rapport NS/NT augmente $(R=-0,637, P<0,01)$. Nous n'avons pas observé de relation entre les paramètres rhéologiques de la pâte et les autres fractions azotées mesurées (NPT/NT et fractions $\gamma$ ).

\section{Évolution des descripteurs de protéolyse en fonction de l'Aw}

L'analyse en composantes principales (fig 9) sur les principaux paramètres mesurant l'intensité de la protéolyse des petits fromages à 6 semaines nous permet de suivre l'évolution des meilleurs descripteurs de cette protéolyse en fonction de l'Aw des fromages. Les 7 individus projetés sur le 1er plan principal (qui regroupe presque $83 \%$ de l'inertie) sont les 7 individus moyens correspondant aux 7 couples de petits fromages analysés. Leur numérotation dans le plan est fonction de leur te-

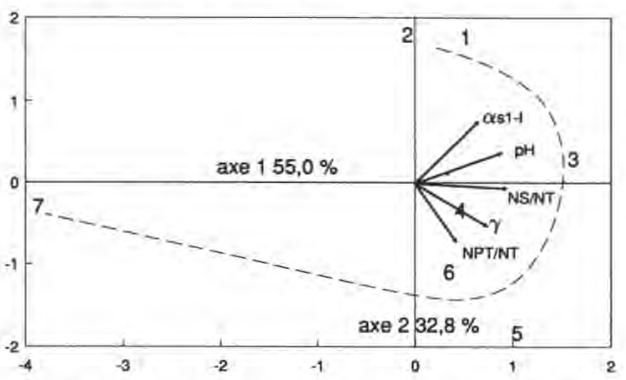

Fig 9. Analyse en composantes principales des fractions azotées. Évolution des descripteurs de la protéolyse en fonction de l'activité de l'eau des fromages.

Principal component analysis of nitrogen fractions. Evolution of the proteolysis descriptors according to water activity of the cheeses.

Variables actives (active variables):

- $\alpha$ s1-I : fraction as1-I ( $\alpha$ s1-I fraction).

- $\gamma$ : fractions $\gamma$ ( $\gamma$ fractions).

- $\mathrm{pH}: \mathrm{pH}(\mathrm{pH})$.

- NS/NT: NS/NT (WSN/TN).

- NPT/NT : NPT/NT (PTASN/TN). neur en $\mathrm{NaCl}$, du moins salé au plus salé. On y voit très clairement que les fromages peu salés (1 et 2) sont caractérisés par les plus grandes teneurs en fraction $\alpha$ s $1-1$, une Aw élevée et une bonne cohésion de pâte. Puis lorsque la teneur en $\mathrm{NaCl}$ augmente, le $\mathrm{pH}$ passe par un maximum (3) puis c'est au tour du rapport NS/NT, des fractions $\gamma$ et enfin du rapport NPT/NT $(4,5,6)$. Pour les fromages très salés (7), le niveau de protéolyse global est faible et pas différencié. De cette manière, on met facilement en évidence qu'en fonction de la teneur en sel, ou plus précisément de l'Aw, ce ne sont pas les mêmes systèmes enzymatiques qui sont responsables de lintensité de protéolyse.

\section{CONCLUSION}

Cette étude a permis de montrer que modifier la teneur en sel d'un fromage est un moyen efficace pour jouer sur l'activité de l'eau. En particulier, fabriquer des petits fromages à partir d'un Gruyère de taille réelle, les saumurer puis les affiner sous film plastique permet d'avoir des fromages homogènes, représentatifs d'une pâte pressée cuite. L'extrait sec de ces fromages évolue peu en 6 semaines d'affinage et lorsque le temps de saumurage varie de 0 à $24 \mathrm{~h}$, l'activité de l'eau semble directement fonction de la teneur en $\mathrm{NaCl}$ dans I'humidité des fromages (0-4\%).

Ainsi, nous avons mis en évidence que dans les conditions de milieu d'un fromage type Gruyère, la nature et lintensité de la protéolyse ainsi que la texture dépendent de l'activité de l'eau en liaison avec la teneur en sel. Celle-ci modifie fortement la protéolyse primaire des caséines mais n'a que peu d'incidence sur la protéolyse secondaire. Nous montrons clairement que l'activité du système plasmine, mesurée par la quantité de fractions $\gamma$ produite dé- 
pend étroitement de l'activité de l'eau du fromage. Elle est maximale pour une valeur d'activité de l'eau correspondant à environ $2 \%$ de $\mathrm{NaCl}$ dans l'humidité du fromage. La dégradation de la caséine $\alpha$ s 1 diminue constamment avec la baisse d'activité de l'eau induite par l'augmentation de sel. Les fromages moins salés, à activité de l'eau élevée, à forte dégradation de la caséine $\alpha s 1$ sont moins fermes et ont une cohésion plus faible.

\section{REMERCIEMENTS}

Ce programme a reçu une aide financière de la région franche-comté (Contrat 3202A). Nous remercions également $\mathrm{J}$ Millet de l'École nationale d'industrie laitière à Poligny (39800) qui a supervisé la fabrication du Gruyère ayant servi de matière première à l'étude, ainsi que $\mathrm{G}$ Duboz et RM Gomez pour leur contribution technique à l'étude.

\section{RÉFÉRENCES}

Adda J, Gripon JC, Vassal L (1982) The chemistry of flavour and texture generation in cheese. Food Chem 9, 115-129

Berdagué JL, Grappin R (1987) Affinage et qualité du gruyère de Comté. II. Influence de l'affinage sur l'évolution des caractéristiques physico-chimiques des fromages. Lait 67, 237-247

Buchet C (1987) Essai de quantification des caséines de fromage à pâte pressée cuite séparées par électrophorèse sur gel PAGE. Etude ITG

Chamba JF (1988) Salage de l'Emmental. I. Influence des conditions de saumurage sur l'absorption du sel et sa cinétique. Lait 68, 121-142

Collin JC, Berdagué JL, Dognin-Bergeret $M$, Grappin R (1987) Affinage et qualité du gruyère de Comté. IV. Étude de la protéolyse. Lait 67, 299-318
Courroye M (1987) L'indice d'affinage : un nouveau moyen de suivre la protéolyse des fromages à pâte pressée cuite par cryoscopie. Ind Alim Agric 104, 169-173

Creamer LK, Olson NF (1982) Rheological evaluation of maturing Cheddar cheese. J Food Sci 47, 631-636, 646

De Jong $L$ (1977) Protein breakdown in soft cheese and its relation to consistency. 2 . The influence of rennet concentration. Neth Milk Dairy J 31, 314-327

Eberhard P (1985) Rheologische Eigenschaften ausgewälter Käsesorten. Thesis, Zürich

El Soda M, Desmazeaud MJ (1982) Les peptides hydrolases des lactobacilles des groupes thermobacterium. I. Mise en évidence de ces activités chez Lactobacillus helveticus, Lactobacillus acidophilus, Lactobacillus lactis, Lactobacillus bulgaricus. Can J Microbiol 28, 1181-1188

Fils-Lycaon B, Buret M, Drouet A, Hartmann C, Duprat $F$ (1988) Ripening and overripening of cherry fruit: use of principal component analysis to check fruit picking and sampling method pertinence and select the most discriminant analysis criteria. Sci Alim 8, 383-396

Fox PF, Walley BF (1971) Influence of sodium chloride on proteolysis of casein by rennet and by pepsin. J Dairy Res 38, 165-170

Garnot $P$, Mollé D (1987) Heat-stability of milkclotting enzymes in conditions encountered in Swiss cheese making. J Food Sci 52, 7577,87

Godinho M, Fox PF (1982) Ripening of Blue cheese. Influence of salting rate on proteolysis. Milchwissenschaft 37, 72-75

Gripon JC, Desmazeaud MJ, Le Bars D, Bergère $\mathrm{JL}$ (1975) Étude du rôle des microorganismes et des enzymes au cours de la maturation des fromages. II. Influence de la présure commerciale. Lait 55, 502-516

Hardy J (1979) L'activité de l'eau, le sel et les moisissures des fromages. Rev Lait Fr 377, 19-25

Heiss E (1961) Essai de dosage de la matière grasse dans le fromage. Dtsch Molk Ztg 82, 67-70

Kaminogawa S, Yamauchi K, Miyazama S, Koga $Y$ (1980) Degradation of casein compo- 
nents by acid protease of bovine milk. J Dairy Sci 63, 701-704

Kuchroo CN, Fox PF (1982) Soluble nitrogen in Cheddar cheese. Milchwissenschaft 37, 331335

Landrock AH, Proctor BE (1951) A new graphical interpolation method for obtaining humidity equilibrium data, with special reference to its role in food packaging studies. Food Technol 5, 332-337

Lawrence RC, Creamer LK, Gilles J (1987) Texture development during cheese ripening. $J$ Dairy Sci 70, 1748-1759

Marcos A, Alcala M, Leon F, FernandezSalguero J, Esteban MA (1981) Water activity and chemical composition of cheese. $J$ Dairy Sci 64, 622-626

Matheson AR (1981) The immunochemical determination of chymosin activity in cheese. $N$ Z J Dairy Sci Technol 15, 33-41

Mathlouthi M, Conry M, Jaillant G, Maitenaz PC (1980) Water vapor sorption of Gruyere cheese. Lebensm Wiss Technol 13, 264-268

MICROSTAT ITCF (1984) Service des études statistiques et informatiques. Station expérimentale ITCF, Boignevilles, 91700 Maisse

Mocquot G (1979) Review of the progress of dairy science: Swiss-type cheese. J Dairy Sci $46,133-160$

Mpagana M, Hardy J (1986) Effect of salting on rheological properties of fresh Camembert cheese as measured by uniaxial compression. Milchwissenschaft 41, 210-213

Noomen A (1978a) Activity of proteolytic enzymes in simulated soft cheese (Meshanger type). 1. Activity of milk protease. Neth Milk Dairy J 32, 26-48

Noomen A (1978b) Activity of proteolytic enzymes in simulated soft cheese (Meshanger type). 2. Activity of calf rennet. Neth Milk Dairy J 32, 49-68

Pearce KN (1977) The complexometric determination of calcium in dairy products. $N Z J$ Dairy Sci Technol 12, 113-115

Phelan JA, Guiney J, Fox PF (1973) Proteolysis of casein in Cheddar cheese. J Dairy Res 40, 105-112

Rüegg M, Blanc B (1977) Beziehungen zwischen Wasser-Sorptionsvermogen, Wasseraktivität und Zusammensetzung von Käse. Milchwissenschaft 32, 193-200

Rüegg $M$, Eberhard $P$, Moor U, Flückiger $E$, Blanc B (1980) Beziehungen zwischen Teigbeschaffenheit und Zusammensetzung von Käse. Schweiz Milchw Forshung 9, 3-8

Sanogo T, Paquet D, Linden G (1987) Protéolyse de la caséine en milieu salin : étude de quatre protéases. Sci Alim 7, 385-398

Searles MA, Argyle PJ, Chandan RC, Gordon JF (1970) Lipolytic and proteolytic activities of lactic cultures. XVIIlth Int Dairy Congr $1 E$, 111

Thomas TD, Pearce KN (1981) Influence of salt on lactose fermentation and proteolysis in Cheddar cheese. NZJ Dairy Sci Technol 16, 253-260

Zevaco C, Gripon JC (1988) Properties and specificity of a cell-wall proteinase from Lactobacillus helveticus. Lait $68,393-408$ 